\title{
"Of technical competence, perceived autonomy and relational expertise": Understanding professional identity among nurses working in a stroke unit*
}

\author{
Rudolf Cymorr Kirby P. Martinez, PhD, RN \\ rmartinez@sanbeda.edu.ph \\ San Beda University - College of Nursing \\ Arellano University - Graduate School of Nursing \\ * Lecture presented at $2^{\text {nd }}$ International Neuroscience Nursing Research Symposium, Hilton Anatole, Dallas Texas
}

\begin{abstract}
This paper explored how nurses working in stroke unit conceptualized and make meaning their professional identity as reflected in their mundane everyday experience at the bedside. Grounded on focused ethnography, seven (7) informants from two stroke unit from two selected hospitals within Metro Manila were chosen to be informants in this study. Participants were selected based on the criteria that (1) They are currently working as nurses in the stroke unit with at least five years of exposure in the said area and (2) they are fluent in English and Tagalog and are willing to participate in this study. Interviews and story-telling sessions were done to gather the necessary narratives which were then analysed via the process of thematization. After the process of analysis, three themes were identified and are as follows: (1) Technical proficiency as a sign of competence; (2) Perceived autonomy as a mark of dignity; (3) Relational expertise as a manifestation of seniority. The themes show in general how technical competence, perceived autonomy and relational expertise were seen by the informants as distinctive marks of their professional identity as nurses working in a stroke units. Insights from this study reveal the unique appreciation of nurses working in stroke unit of their concept of competence, autonomy and expertise. The said concepts are deeply ingrained in their everyday lives that it has become part of their disciplinary hallmarks. Since the ultimate aim of focused ethnography is not to produce a universal truth that can be applied to all context and setting but rather provide insights on specific context sensitive phenomena that will serve as a springboard for the improvement of the context where the insights were gleaned upon from, the following implications were proposed: Professional updates, trainings and preceptorship program that focus on technical competencies, communication skills, and conflict management techniques are suggested. Further, empowerment and inter professional bonding activities, such as open forums might also be beneficial in the context of collegiality and collaborative practice.
\end{abstract}

Martinez, R. C. K. P. (2018). "Of technical competence, perceived autonomy and relational expertise": Understanding professional identity among nurses working in a stroke unit. SocArx. Doi: 10.31235/osf.io/dh65f 


\section{Context of the Study}

Stroke continues to be the one of the global cause of mortality (WHF, 2017). In the Philippines alone, latest available data shows that it still remain to be the second leading cause of death in 2012 (WHO). With the global burden of disease that stroke continues to produce, trends in the development of specialty areas that caters to stroke patients have been increasing in the recent years. Abroad, as early as 1990's, stroke units have been created by medical institution to specifically cater to patients suffering from stroke (Dennis and Langhorne, 1994). Studies have continually shown the effectiveness of stroke in terms of financial benefit (Sheppard and Ko, 2009), patient outcomes (Jammali-Blasi et al, 2011, Walter et al, 2009), mortality reduction (Candelise, 2007; Langhorne et al, 1993), length of stay (Chen, McClaran, Buchan, 2008). Locally, the Philippines is following this trend of creating specialized unit for select group of patient. One of the hospitals that have recently created a stroke unit is the Western Visayas Medical Center.

Although the effectiveness of the stroke unit vis-à-vis, the necessity of creating one have been explored through research abroad (Evans et al, 2001; Jammali-Blasi et al, 2011; Langhorne, de Villiers, Pandian, 2012; Martinez-Sanchez et al, 2010; Sheppard and Ko, 2009), no local study have been made to understand how the stroke unit, through its nurses, functions and make meaning of their experience in it. Studies have shown the central importance of nurses in the stroke units (Clarke, 2014) such that their appreciation and meanings they attach to the stroke unit inevitably affects the functioning of the unit per se (Barreca and Wilkins, 2008; Burton, Fisher and Green, 2009; Clarke and Holt, 2015; Hart, 1998; Pound and Ebrahim, 2000; Seneviratne, Mather and Then, 2009). Understanding the life-world of these nurses therefore, would enable managers, administrators and practitioners to effectively create a facilitative atmosphere conducive to working and in effect elevate the quality of care in the unit as a whole.

The continued global burden of stroke, increasing creation of stroke unit and lack of local studies in the nurses' appreciation of the stroke unit justify the gap by which this study intends to fill. Specifically, this paper tackles how professional identity was conceptualized and comes into play within the context of nurses working in the stroke units. 


\section{Methodology}

This study is rooted on the interpretive philosophy, specifically focused ethnography as its vantage point of making sense of the world of nurses working in the stroke unit. Reimer as cited by Lapan et al (2011) defines ethnography as study of one particular group or phenomenon, documenting the practices and belief of those within the group from their own perspective. Simply put, it is the study of a particular group where culture, the "total pattern of human behavior and its products embodied in speech, action, and artifacts and dependent upon man's capacity for learning and transmitting knowledge to succeeding generations" (Webster, 1993) is the main glue that holds the group together. As Arnould (1998) puts it, ethnography aims to explain the ways their shared system of meanings, i.e. culture and its people co-construct each other as reflected by the behavior and experiences of each member. Moreover, Hammersley \& Atkinson (1995) opinioned that ethnography can be utilized in three ways; to elicit a cultural knowledge, to holistically analyze a society and to understand social interaction and meaning making.

Specifically, focused ethnography was utilized as the lens by which the lives of the nurses working in stroke unit were understood. Focused ethnography is a subfield of ethnography which specifically deals with a specific problem within a specific context of a culture (Knoblauch, 2005; Morse \& Richards, 2002). In focused ethnography, culture and subcultures are understood as universal and unbounded (Mayan, 2009), the reason why it is utilized to study phenomenon of highly specialized fields of study (Cruz \& Higginbottom, 2013; Knowblauch, 2005).

Criterion sampling based on theoretical saturation was utilized to ascertain the breadth and depth of the gathered narratives from the informants. Seven (7) registered nurses, recruited via snowball and referral technique, working in two selected stroke units were chosen based on the criteria that they have been working as a nurse in the stroke unit for at least 5 years, fluent in English and Filipino and are able to fully articulate their experiences. Narratives from the informants were gathered via multi phased, face to face, in-depth interview in a place they specifically chosen during their day-off from their hospital duty. Interviews last at least 60 minutes per session with the longest interview lasting for at least 2.5 hours. A total of 15 interviews were done with continuous online correspondence with the informants to clarify points and themes resulting from the analysis of their experiences. 
The process of thematization rooted on interpretive paradigm developed by the researcher was utilized to analyze the narratives from the informants. Figure 1 shows the totality of the process.

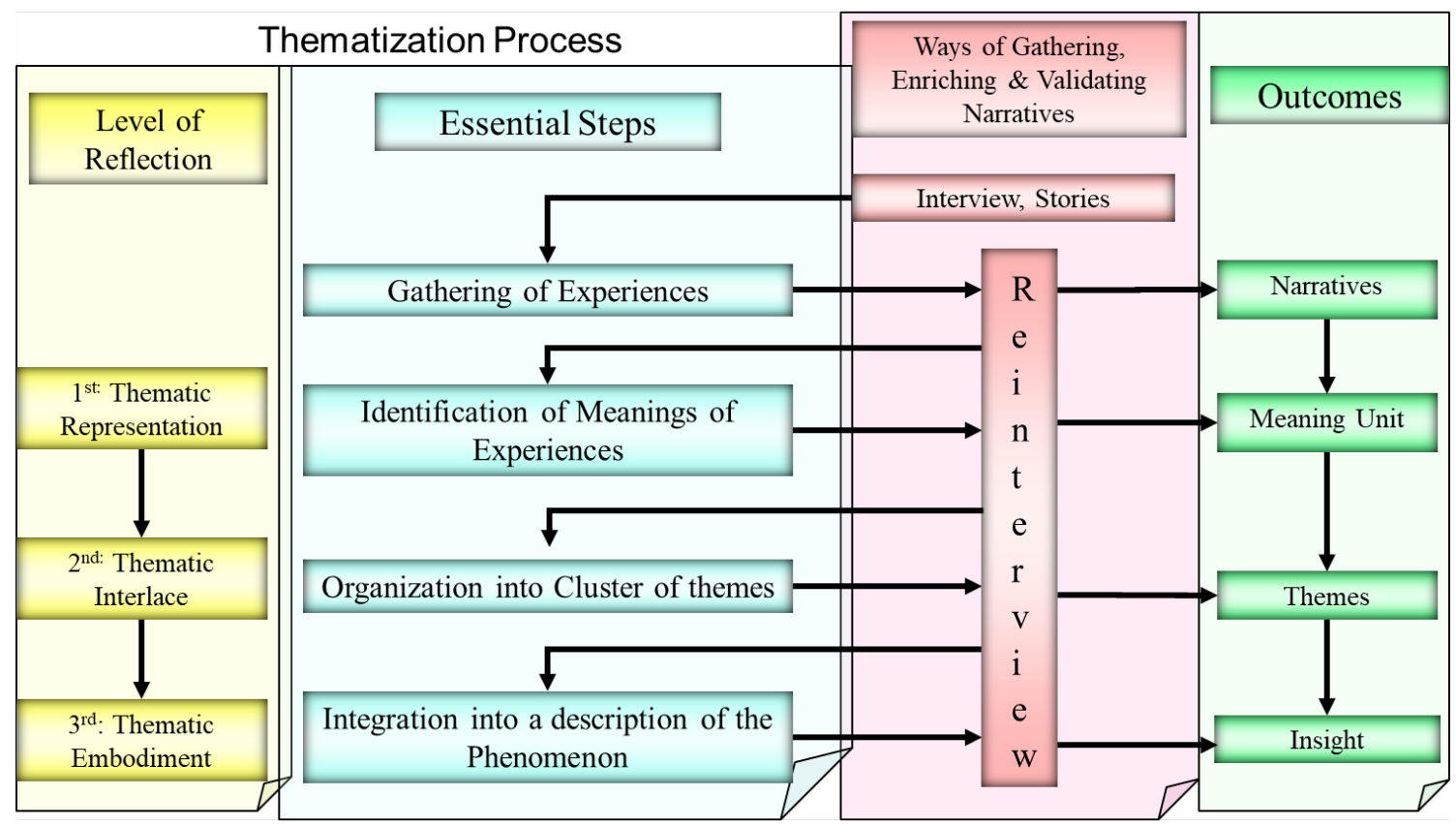

Specifically, the following process describes and summarizes how the experiences were reflectively analyzed in this study. Specifically, it shows the process of reflective analysis of the experiences, together with its concurrent level of reflection, essential steps, ways of enriching the experiences and their outcomes (Figure 1).

The yellow shaded boxes shows the level of reflections that was utilized in this study, opposite of which, blue shaded boxes, are the essential steps in the reflective analysis related with each level. In addition to that, the pink shaded boxes represent the ways on how the narratives was enriched, validated and counter-validated, by the informants' experiences. The green shaded boxes represent the outcomes of each essential steps as well as each level of reflections.

The following are the essential steps in the reflective analysis of the informants' experiences

1. Initial interview and stories from the participants were gathered after which they were encoded verbatim. This represents the first essential steps termed as the "gathering of experiences". Thereafter, the encoded narratives were given back to informants to validate 
their content which then resulted in the narrative, a validated account of the participant's experiences and will be thus considered as the "raw data".

2. After the transcription, the researchers then identified the essential meanings found in these narratives via identifying their thought markers. As a result of this first reflection, the enhanced and validated thought element of the experiences was produced. During this phase, member checking of the thought elements were performed both to maintain the rigor of the study as well as ascertain its credibility. The end goal is to produce a single version of the narratives with thought elements agreed upon by the informants.

3. Next, after the meaning units of the combined experiences were explicated, the researcher then reflected on these thought elements to give produce the themes, representing the second level of reflection, the thematic interlace. The resulting themes were again validated and counter validated by the informants through an interview which then results in the enriched and validated themes.

4. To embellish grounding to the significant insights gathered from the process of reflective analysis, reflective resonance was done by situating the resulting themes from literatures. This process, paralleling the related literature review done in a quantitative paradigm, will neither affirm nor negate the insights formed from the reflection but merely add a lens by which the result can be appreciated, following the philosophical underpinning of a contextual understanding based on the tradition of the qualitative philosophy. This constitutes the 3rd level of reflection, the thematic embodiment.

The process of validation and counter validation was done by presenting the significant outcomes of each step in the process of reflective analysis to the informants for resonance. It is only when they have agreed that the outcomes mirrors their narratives shall the researchers continue to the next level of reflective analysis. This continuous process of validation and counter validation ascertain that great emphasis is placed on the primacy of the participants' narratives as well as ground the study on the tenet that understanding is a co-created reality between the researchers and the participants.

Moreover, prior to the conduct of this study, an IRB clearance was secured from the San Beda Ethics Council. The following ethical considerations were ascertained during the conduct of this study:

Martinez, R. C. K. P. (2018). "Of technical competence, perceived autonomy and relational expertise": Understanding professional identity among nurses working in a stroke unit. SocArx. Doi: 10.31235/osf.io/dh65f 
1. Prior to the study, the researchers obtained an ethics clearance from the Ethics Review Board to ensure that the study will be done within the parameters of an ethical study.

2. Verbal and written consent was also obtained from the informants, indicating that they are fully aware of their involvement and its voluntary nature. The informants was be given the right to refuse and/or withdraw from the study at any time if they deemed necessary without any form of penalty or repercussion.

3. The informants were likewise be given the study's goal and objective, associated risk, and benefits of participation. Coercion, in any form, was never utilized in the process of informants recruitment. Further, they were informed that the interview will be done multiple times and they have the right to choose where it would be conducted.

4. Further, the possibility of this paper to be presented and/or published was made known to the informants. Strict compliance to the process of maintaining their anonymity was adhered such that no traceable information leading to their identification was disclosed.

5. During the course of this study, each informants was given pseudo names in order to provide anonymity and maintain confidentiality. The identities of the informants were only known by the researcher. Further, no other data except for age and length of experience will be gathered from the informants. The name of their institution remained unanimous all throughout the research process and result dissemination.

6. In the event that they made mention the names of their institution or any data that might reveal their identities (e.g. nicknames, etc) during the tape recorded interviews, these data was not included in the verbatim transcription of the narratives. Moreover, access to the tape recorded interviews was only available to the researcher.

7. All the gathered data from the informants was kept secured and accessible only to the researcher and will be destroyed through appropriate means (e.g. shredding) after 5 years.

8. Post interview processing was also done for each informant at the end of every interview session. In the event that the informant felt a need for counseling, a registered guidance counselor was provided to them free of charge. No informant availed of such counseling.

9. Adhering to the principles of qualitative paradigm and the primacy of the informant's experience, continued validation and counter validation was done at each significant step of the process. This was done by going back to the informant $\mathrm{s}$ for the accuracy of the 
analytical process ensuring that the informants take an active role in meaning making as well maintaining the authenticity of their experiences

\section{Findings and Discussion}

Seven (7) informants working in two selected stroke unit from two different tertiary hospitals within Metro Manila participated in this study. All informants were practicing nurses stationed in stroke units for at least 5 years with age range of $23-45$ years old. The stroke units they are working with are specialized unit within the hospital system working directly under the supervision of a physician.

Analysis from their narratives specifically focuses on how their everyday experience working as a nurse in the stroke unit affects their view on their professional identity within their intra and inter-professional relationships. After careful analysis of their narratives the following patterns vis-à-vis themes were gleaned upon: (1) Technical proficiency as a sign of competence; (2) Perceived autonomy as a mark of dignity; (3) Relational expertise as a manifestation of seniority.

\section{Technical proficiency as a sign of competence}

Technical proficiency, in terms of technological adeptness and aptitude in procedural nursing skills, were seen by the informants as a sign of competence. For the informants, there exist a consensus that the stroke unit is a

\section{"place where machine and people co-exist". (Informant 4)}

The everyday activities in the stroke unit consist of receiving the patient from the outgoing shift, assessment of the patients assigned, carrying out physician's orders, caring for the patients and answering queries from relative, physicians, and other health care practitioner in between activities. The basic set-up of the stroke unit consists of the nurses' station, a lounge for eating, patient's room and a variety of contraptions surrounding the patient. Informant 2 summed up the set-up by saying 
"There's the mechanical ventilator, infusion pump, kangaroo pump, different kinds of pump, ECG, Vital sign monitor, O2 saturation monitor and some other machines that we need to know how to operate. We must know how to operate"

It is no wonder that for the informants, technical proficiency in terms of mechanical adeptness in knowing how to use machines is seen as a sign of competence. Much of the interaction of the informants with patients in stroke units involves the use of some form of technology, from the simple manual taking of vital signs to the more complex mechanical ventilator. Communication with the patient and their relatives are initiated most of the time during procedures involving machines and technology. Informant 1 exemplifies this by saying:

"We are very toxic in the unit that most of the time, we cannot seat and talk with our patients anymore. Also, most of them are non-responsive so we talk to them when we are doing procedures as if they are conscious. That's the only time we mostly interact, talked with them"

Another aspect of technical proficiency labeled by the informants as a sign of competence is their aptitude in procedural nursing skills, specifically their "assessment skills". All of the informants take great pride in narrating how their assessment skill is "different, better and unique" compared to the other nurses. By assessment skills, they mean the complex assessment skills like neurological and functional assessment. It seems that for them, without "a more than the normal" aptitude in these skills, one cannot claim themselves as a stroke nurse.

"Our assessment skills (neurological assessment) is different... I cannot pinpoint how different but we know it is different since when we are pulled out from the unit to a different floor, the other nurses would always comment things like "ahhh, that's why (he is OC in assessment) because he is a stroke nurse" (Informant 2) 
Informant 5 would further point out that

"You are not a stroke nurse unless you know your assessment by heart. That is our bible. Even if you transfer a nurse from stroke unit to the other ward, you will know he/she is a stroke nurse just by the(ir) assessment"

For the informants, technical proficiency, both in technology and skills, serves both as a mean of facilitating the provision of nursing care and an identifying hallmark of their unique identities as stroke nurses.

This idea of technical proficiency as a part of the structured competency among health care practitioners working in stroke unit is supported by the study done by Clarke \& Holt (2014) on understanding nursing in a stroke unit.

\section{Perceived autonomy as a mark of dignity}

Perceived autonomy, in terms of independent decision strategy, self-regulation and interprofessional respect, were seen by the informants as marks of professional dignity.

The informants unanimously agreed that they felt they are more empowered when they are practicing as nurses in the stroke unit. This empowerment, as informant 5 verbalized

"comes from our autonomy here (in the stroke unit). We are at par with the physicians... They (fellows) trained us and in return we train them (residents)"

Working in the stroke unit, the informants felt that they were given a free hand to think independently as health practitioners and not as an assistant to the physicians. Prior to the deployment in the stroke unit, the informants were already exposed to other areas in the hospital and are more or less acquainted with the procedural protocols and distinctive nuisance of some physicians. Moreover, they are rigorously trained, ranging from 3 months to 6 months prior to their exposure in the stroke unit. These, coupled with the informants' impression of the monotony and repetitive task in the stroke unit, gave them a sense of autonomy as evident by their independent decision strategies. Informant 2 shares the following incident: 
"There are times when we know what to do with the patient and we just do it and let the physician knows what we did. The carrying out comes first before the order is even written...

Other times, we suggest what can be done because we have memorize the routine orders already"

Informant 2's story is a recurring narrative in the experience of the informants. Other informants shares a different story but with the same theme, that nurses working in the stroke unit can decide independently and are autonomous.

It is also interesting to note that the stroke unit, including the staffs working in it, is under the administrative supervision of a physician. The informants working in the stroke unit is not under the nursing department but rather under "special services". It may seem counterproductive at first but the informants unanimously agreed that this set-up somehow empowers them for a variety of reasons. Informant 4 shares the following snippet of why they prefer to be under the administrative supervision of a physician:

"It's all about the duty schedule. When we were at the floor (under the nursing service), we only have one day off most of the time and requesting for a day off is such a hassle... in the stroke unit, we are given at least 2 days off (because of toxic duty) and requesting for an off is much much easier"

It seems that for the informants, the minimized red tape when it comes to requesting dayoffs in the stroke unit enables them to have a sense of autonomy to somehow have a control over the days when they want to rest. Further, it is worth noting that although the nurses are under the administrative supervision of a physician, they do not meddle in the plotting of duty days by the head nurse of the unit. For the informants, this enables them to self-regulate and further adds to their perceived sense of autonomy in the stroke unit.

Having memorized the routine activities in the stroke unit and an increase confidence as they master their craft, the informants felt that somehow they have gained respect from the other health care provider working in the stroke unit. Having a general feeling of respect somehow increase the informants' sense of self-worth, further increasing their perceived sense of autonomy. Informant 2 shares the following: 
"Yes, the physicians will test you if you really know what you are doing but it is relatively easy to pass, I mean we memorize the routines in the unit and we are the one at the bedside. We know the patient more... There are times that the consultants will ask us "how is the patient" and somehow you will feel "Oh my, I am now worthy"..."

For the informants, their perceived sense of autonomy arising from their feeling of empowerment as independent, self-regulating and respect-worthy health care providers serves as an emblem of their professional stature and self-determination.

The notion that respect and support from senior nurses and physicians boost the nurses' confidence is shared by the result of the research done by Catangui \& Robers (2014) on the lives of nurses in one hyper-acute stroke unit.

\section{Relational expertise as a manifestation of seniority}

Relational expertise, in terms of conflict management and smooth interpersonal relation with people, were seen by the informants as a manifestation of seniority.

For the informants, the concept of "seniority" encompasses having both wisdom and expertise. Although the element of age, as it relate to one's length of employment as a nurse, still exist among the informants; the idea that relational expertise as the implied transitionary element from being a novice to that of an expert is implied in their narratives.

Specifically, for them, to be a senior nurse means one can instinctively decide on conflicts and can interact with people with ease and confidence. When asked when she felt that she has become a senior nurse, informant 7 shares the following:

"It's hard to pinpoint actually... it just happen, I mean when the people around you sees you as a senior and you feel you are already a senior nurse maybe that's the time?... You see, senior (nurses) in the stroke unit exemplify the adage "grace under pressure" I guess... They know when to talk and when not to"

It must be noted that in the stroke unit, there exist only the staff nurse and head nurse position. For the informants, seniority is not a position granted by the administration but an 
implied role based on one's ability to relate with people. Although the role is implied, the functions of a senior nurse is multi-faceted but can be summed up as "maintenance of harmony". It is for this reason that the informants agreed that relational skill and expertise is a core characteristic of a senior nurse. Informant 1 shares the following:

"I am not yet a senior, I do not feel one yet though I am working there for a long time... (why?!) Because I cannot handle conflict that easily and I am not confident to lead my fellow nurses, maybe someday but not now"

For the informants, relational expertise as expressed by skilled conflict management and smooth interpersonal relations with people is a manifestation of seniority. Their concept of seniority is closely knit with their conceptualization of professional identity and maturity.

Farnell \& Dawson (2006) research on the experience of nurses new to critical care, also suggest that effective preceptorship facilitate socialization which enable nurses to progress from novice to advance beginner.

\section{Insights and Implications}

The ultimate aim of focused ethnography is not to produce a universal truth that can be applied to all context and setting but rather provide insights on specific context sensitive phenomena that will serve as a springboard for the improvement of the context where the insights were gleaned upon from. Based on the findings of this research, the following are implied:

1. Since technical proficiency was seen by the informants as a sign of competence, trainings focusing on technological and procedural mastery, specifically assessment skills, should be continued especially for new nurses entering the stroke unit.

2. Training via a formal preceptorship program from a more experienced might be helpful in developing decision making strategies for novice nurses working in stroke unit.

3. Self-regulation, as evident by empowering nurses by allowing them to suggest their dayoff should be continued as it increases their perceived autonomy, self-determination and job satisfaction. 
4. Inter-professional respect was seen as a mark of professional integrity thus activities to promote camaraderie, such as team building activities, might be implied. Further, open forum during meetings could also facilitate early resolution of beginning interprofessional conflicts.

5. There seem to be a consensus among the informants that they lack relational expertise as they perceived it developing not by training but by experience. Senior-junior buddy system could facilitate in the development of relational skills among novice nurses working in stroke unit. Moreover, trainings focusing on soft skills, like handling conflict or communication techniques, could be given as part of pre-deployment coaching or professional updates

\section{References}

Allmark, P.J., Boote, J., Chambers, E., Clarke, A., Mcdonell, A., Thompson, A., \&Tod, A. (2009). Ethical issues in the use of in-depth interviews: literature review and discussion. Research Ethics Review, 5 (2), 48-54.

Arnould, E.J. (1998), Daring consumer-oriented ethnography, in stern, b. (Ed.), Representing consumers: voices, views and visions. London:Routledge

Barreca, S., Wilkins, S. (2008). Experiences of nurses working in a stroke rehabilitation unit. Journal of Advanced Nursing, 63(1), 36-44. doi: 10.1111/j.1365-2648.2008.04648.x

Burton, C., Fisher, A., Green, T. (2009). The organisational context of nursing care in stroke units: a case study approach. International Journal of Nursing Studies, 46(1), 86-95. doi: 10.1016/j.ijnurstu.2008.08.001

Candelise, L., Gattinoni, M., Bersano, A., Micieli, G., Sterzi, R., Morabito, A. (2007). Strokeunit care for acute stroke patients: An observational follow-up study. The Lancet, 369, 299-305. doi: 10.1016/S0140-6736(07)60152-4

Catangui, E.J. \& Roberts, C.J. (2014). The lived experiences of nurses in one hyper-acute stroke unit. British Journal of Nursing, 23(3), 143-148. Doi: /10.12968/bjon.2014.23.3.143

Chen, L., McClaran, J., Buchan, A. (2009). Impact of acute stroke unit on hospital length of stay. Archives of Gerontology and Geriatrics, 49(1), 12-15. doi: 10.1016/j.archger.2008.07.008

Clarke, D. (2014). Nursing practice in stroke rehabilitation: systematic review and metaethnography. Journal of Advanced Nursing, 23(9-10), 1201-1226. doi: $10.1111 /$ jocn.12334

Clarke, D., Holt, J. (2015). Understanding nursing practice in stroke units: a Q-methodological study. Disability and Rehabilitation, 37, 1870-1880. doi: 10.3109/09638288.2014.986588

Cruz, Edward V. \& Higginbottom, Gina (2013). The use of focused ethnography in nursing research. Nurse Researcher, 20(4), 36-43. doi: 10.7748/nr2013.03.20.4.36.e305

Dennis, M., \& Langhorne, P. (1994). So stroke units save lives: where do we go from here? BMJ : British Medical Journal, 309, 1273-1277. 
Evans, A., Perez, I., Harraf, F., Melbourn, A., Steadman, J., Donaldson, N., Kalra, L. (2001). Can differences in management processes explain different outcomes between stroke unit and stroke-team care?. The Lancet, 358, 1568-1592. doi: 10.1016/S0140-6736(01)066521

Farnell, S. \& Dawson, D. (2006). 'It's not like the wars'. Experiences of nurses new to critical care: A qualitative study. International Journal of Nursing Studies, 43, 319-331. Doi: 10.1016/j.ijnurstu.2005.04.007

Guion, L., Diehl, D., and McDonald, D. (2011). Conducting an in-depth interview, University of Florida. Retrieved from http://edis.ifas.ufl.edu/fy393

Hammersley, M., \& Atkinson, P. (1995). Ethnography: Principles in practice. London: Routledge. Chicago

Hart, E. (1998). Evaluating a pilot community stroke service using insights from medical anthropology. Journal of Advanced Nursing, 27(6), 1177-1183. doi: 10.1046/j.13652648.1998.00646.x

Jammali-Blasi, A., McInnes, E., Markus, R., Faux, S., O’Loughlin, G., Dale, S., Middleton, S., (2011). A study of 90-day outcomes for a cohort of patients admitted to an Australian metropolitan acute stroke unit. Journal of Vascular Nursing, 29(1), 3-10. doi: 10.1016/j.jvn.2010.11.004

Knoblauch, H. (2005). Focused Ethnography. Forum Qualitative Sozialforschung / Forum: Qualitative Social Research, 6(3). doi:http://dx.doi.org/10.17169/fqs-6.3.20

Langhorne, P., de Villiers, L., Pandian, J., (2012). Applicability of stroke-unit care to lowincome and middle-income countries. Lancet Neurology, 11(4), 341-348. doi: 10.1016/S1474-4422(12)70024-8

Langhorne, P., Williams, B., Gilchrist, W., Howie, K. (1993). Do stroke units save lives?. The Lancet, 342, 397-398. doi: 10.1016/0140-6736(93)92813-9

Lapan, S., Reimer, F., Quartaroli, M. (Eds) (2011). Qualitative research: An introduction to methods and designs. USA: Lippincot Williams and Wilkins

Martinez, R.C.K.P. (2013). Hinabingugnayan: nature of caring among filipino nurses with children. (Unpublished doctoral dissertation). Asian Social Institute, Manila, Philippines.

Martínez-Sánchez, P., Fuentesa, B., Medina-Báez, J., Grande, M., Llorenteb, C., Parrilla, P., Fusterc, A., Gil, A., Sánchez, M., Olguína, C., García-Caballero, J., Díez-Tejedo. E. (2010). Development of an acute stroke care pathway in a hospital with stroke unit. Neurologia, 25(1), 17-26. doi: 10.1016/S2173-5808(10)70004-3

Mayan, Maria (2009). Essentials of qualitative inquiry. Walnut Creek, CA: Left Coast Press.

McKevitt, C., Redfern, J., Mold, F., \& Wolfe, C. (2004). Qualitative studies of stroke: A systematic review. Stroke: Journal of the American Heart Association, 35(1), 14991505. doi: 10.1161/01.STR0000127532.64840.36

Morse, Janice M. \& Richards, Lyn (2002). Readme first for a user's guide to qualitative methods. Thousand Oaks, CA: Sage.

Munhall, P. L. (2007). Nursing research: A qualitative perspective. Sudbury, Mass: Jones and Bartlett.

Parrish, P. (2006). Design as storytelling. TechTrends,50(4), 72-81. doi:10.1007/s11528-006-0072-7.

Pound, P., Ebrahim, S. (2000). Rhetoric and reality in stroke patient care. Social Science \& Medicine, 51(10), 1437-1446. doi: 10.1016/S0277-9536(00)00040-X 
Sammel, A. (2003). An invitation to dialogue: Gadamer, Hermeneutic phenomenology, and Critical environmental education. Canadian Journal of Environmental Education, 8(1), 155-168.

Salter, K., Hellings, C., Foley, N., \&Teasell, R. (2008). The experience of living with stroke: a qualitative metasynthesis. Journals of Rehabilitation Medicine, 40(8), 595-602. doi: 10.2340/16501977-0238

Seneviratne, C., Mather C., Then K. (2009). Understanding nursing on an acute stroke unit: perceptions of space, time and interprofessional practice. Journal of Advanced Nursing, 65(9), 1872-1881. doi: 10.1111/j.1365-2648.2009.05053.x

Shank, G. (2002).Qualitative Research.A Personal Skills Aproach. New Jersey: Merril Prentice Hall.

Sheppard, L. and Ko, K. (2009). A new stroke unit: implementation issues. Hong Kong Physiotherapy Journal, 27(1), 7-10. doi: 10.1016/S1013-7025(10)70003-8

Stahlke Wall, S. (2014). Focused Ethnography: A Methodological Adaptation for Social Research in Emerging Contexts. Forum Qualitative Sozialforschung / Forum: Qualitative Social Research, 16(1). doi:http://dx.doi.org/10.17169/fqs-16.1.2182

Walter, A., Seidel, G., Thie, A., Raspe, H. (2009). Semi-intensive stroke unit versus conventional care in acute ischemic stroke or TIA--a prospective study in Germany. Journal of Neurological Science, 287(1), 131-137. doi: 10.1016/j.jns.2009.08.010

Martinez, R. C. K. P. (2018). "Of technical competence, perceived autonomy and relational expertise": Understanding professional identity among nurses working in a stroke unit. SocArx. Doi: 10.31235/osf.io/dh65f 